\title{
ATIVIDADE ACARICIDA DOS ÓLEOS ESSENCIAS DE FOLHAS E FRUTOS DE Xylopia sericea SOBRE O ÁCARO RAJADO (Tetranychus urticae KOCH)
}

\author{
Wendel José Teles Pontes, José Cândido Selva de Oliveira e Cláudio Augusto Gomes da Câmara* \\ Departamento de Química, Universidade Federal Rural de Pernambuco, Av. Dom Manoel de Medeiros s/n, 52171-900 \\ Recife - PE, Brasil \\ Manoel Guedes Correia Gondim Júnior e José Vargas de Oliveira \\ Departamento de Agronomia, Universidade Federal Rural de Pernambuco, Av. Dom Manoel de Medeiros s/n, 52171-900 \\ Recife - PE, Brasil \\ Manfred Oswald Erwin Schwartz \\ Departamento de Química Fundamental, Universidade Federal de Pernambuco, 50740-540 Recife - PE, Brasil
}

Recebido em 13/4/06; aceito em 30/8/06; publicado na web em 26/3/07

\begin{abstract}
ACARICIDAL ACTIVITY OF the ESSENTIAL OILS OF LEAVES AND FRUITS OF Xylopia sericea St. Hill. ON THE TWO SPOTTED SPIDE MITE (Tetranychus urticae Koch). The essential oils of the leaves and fruits obtained by hydrodistillation of Xylopia sericea, collected in the restinga area of Pernambuco, were analyzed by GC (HP 5890 SERIES II) and GC/MS (HP 5890B SERIES II/ MSD 5971). A major part of the volatile components identified in the oils of fruits and leaves were monoterpenes and sesquiterpenes. Cubenol (57.43\%) and $\alpha$-epi-muurolol (26.09\%) were the main compounds found in the leaves, whereas $\beta$-pinene (45.59\%) and $\alpha$-pinene (17.18\%) were the fruits major components. The acaricidal activity of the essential oils was evaluated for Tetranychus urticae. The oil of the leaves was more active than that of the fruits showing an $\mathrm{LC}_{50}$ value of $4.08 \mu \mathrm{L} / \mathrm{L}$ of air for a $72 \mathrm{~h}$ period.
\end{abstract}

Keywords: Xylopia sericea; essential oil constituents; Tetranychus urticae.

\section{INTRODUÇÃO}

A procura por novas espécies vegetais com atividade inseticida tem aumentado nos últimos anos, devido aos problemas ambientais causados pelo uso indiscriminado de inseticidas sintéticos na proteção de culturas em todo o mundo. Como resultado, vários estudos levaram à identificação de plantas inseticidas e à caracterização de seus respectivos compostos bioativos ${ }^{1}$. Dentre os compostos naturais comprovadamente bioativos, destacam-se o piretro, nicotina, rotenona e azadiractina, isolados a partir das espécies Chrysanthemum cinerariafolium (Trev.), Nicotiana tabacum L., Derris sp. e Azadirachta indica A. Juss., respectivamente ${ }^{1}$.

Das inúmeras espécies vegetais utilizadas, com sucesso, no controle de pragas agrícolas, algumas são pertencentes à família Annonaceae $^{2}$. Essa família compreende cerca de 130 gêneros e aproximadamente 2300 espécies, distribuídas em regiões tropicais e subtropicais na África, Ásia, Austrália, América Central e do Sul ${ }^{3}$. No Brasil, esta família está bem representada com aproximadamente 27 gêneros e 290 espécies, sendo que o gênero Xylopia é um dos maiores com mais de 150 espécies, amplamente distribuídas em diversas partes do mundo. No Brasil, esse gênero é típico do cerrado, embora ocorra em ambientes com estratos arbustivo e arbóreo mais densos.

Muitas espécies do gênero Xylopia têm sido estudadas quanto à caracterização de sua composição química, identificando-se alcalóides, compostos voláteis, flavonóides, terpenóides e esteróis ${ }^{3-6}$.

O gênero Xylopia é reconhecido por apresentar propriedades medicinais, dentre as quais destacam-se as atividades antimicrobiana $^{7,8}$ e citotóxica ${ }^{9}$. Como a busca por propriedades inseticidas em plantas medicinais tem crescido nos últimos anos ${ }^{10}$, a literatura tem relatado estudos onde o potencial acaricida, inseticida e nematicida de espécies do gênero Xylopia é avaliado quanto a sua

*e-mail: camara@ufrpe.br eficácia no controle de pragas agrícolas ${ }^{2}$. O extrato hexânico de frutos de $X$. aethiopica apresentou forte atividade antialimentar sobre operários de cupins da espécie Reticulitermes speratus Kolbe $^{11}$. Separações cromatográficas desse extrato resultaram no isolamento de diterpenos derivados do ácido cauranóico como princípios ativos ${ }^{11}$. Recentemente, estudos biológicos voltados para o óleo essencial desta mesma espécie revelou seu potencial inseticida contra pragas de grãos armazenados ${ }^{12,13}$.

Xylopia sericea St. Hill. é uma espécie arbórea, que cresce naturalmente no Nordeste brasileiro, onde é conhecida popularmente como "embiriba". As cascas da madeira são utilizadas na indústria caseira de cordoaria. A infusão de seus frutos é usada popularmente no tratamento de perturbações gástricas, sendo suas sementes carminativas e usadas como condimento, substituindo a pimenta do reino ${ }^{14}$. Investigação prévia dos constituintes químicos desta planta resultou no isolamento de mono e diterpenos a partir das sementes ${ }^{15}$.

Alguns estudos preliminares relacionados com a composição química do óleo essencial dessa espécie foram realizados a partir de diferentes partes do vegetal, como por ex., fruto ${ }^{16-18}$, folha ${ }^{18}$, caule $^{18}$ e raiz ${ }^{18,19}$. Lemos et al. ${ }^{17}$ e Fournier et al. ${ }^{19}$ também investigaram o potencial antimicrobiano do óleo essencial da raiz e do fruto, respectivamente.

Em geral, devido a sua alta volatilidade e possível ação fumigante, os óleos essenciais têm sido investigados quanto ao seu potencial no controle de pragas instaladas em casas de vegetação. $\mathrm{O}$ estudo de propriedades acaricidas do óleo essencial de $X$. sericea é particularmente importante para o controle de ácaros fitófagos, destacando-se, dentre eles, o Tetranychus urticae Koch, popularmente conhecido por ácaro rajado, o qual é considerado praga para inúmeras culturas economicamente importantes em todo o mundo, como o algodoeiro, feijoeiro, morangueiro, mamoeiro, tomateiro e videira $^{20-22}$. Apesar da prévia investigação fitoquímica dos frutos, 
bem como os relatos da ação antimicrobiana e da composição química do óleo essencial da espécie em estudo, que ocorre em diferentes regiões do Nordeste brasileiro, nenhum relato foi feito sobre a composição química e atividade acaricida de seu óleo essencial a partir do fruto e folha, que ocorre em Pernambuco.

Sendo assim, o presente trabalho tem como objetivo determinar a composição química do óleo essencial de folhas e frutos de $X$. sericea, que ocorre na floresta de restinga do litoral sul de Pernambuco, bem como seu efeito fumigante sobre o ácaro rajado, avaliado pelo modelo experimental in vitro de papel de filtro impregnado.

\section{PARTE EXPERIMENTAL}

\section{Material botânico}

Frutos e folhas de $X$. sericea foram coletados, no período da manhã, na área de restinga da praia de Itapuama no litoral sul de Pernambuco, Brasil, em setembro de 2005. A planta foi identificada pela Dra. M. F. de Sales, do Depto. de Biologia da Universidade Federal Rural de Pernambuco (UFRPE) e uma exsicata do material botânico foi depositada no Herbário Vasconcelos Sobrinho da UFRPE, sob o número 48090.

\section{Obtenção dos óleos e análise química por CG e CG/EM}

Folhas e frutos, separadamente, foram submetidos à hidrodestilação por $2 \mathrm{~h}$, utilizando um aparelho tipo Clevenger modificado para obtenção dos óleos. Devido à diferença de densidade, os óleos foram separados da água, secos com $\mathrm{Na}_{2} \mathrm{SO}_{4}$ anidro e armazenados em frascos selados, sob baixa temperatura antes da análise química e de serem usados nos experimentos. Os rendimentos dos óleos foram calculados a partir do peso do material fresco. Todo o procedimento foi realizado em triplicata.

Os óleos obtidos foram analisados por cromatografia gasosa acoplada à espectroscopia de massas utilizando cromatógrafo HP 5890B Series II, acoplado a espectrômetro de massas HP-5971, equipado com uma coluna capilar de sílica fundida J \& W Scientific DB5 (30 m x 0,25 mm x 0,25 $\mu \mathrm{m})$.

A análise cromatográfica (CG) foi obtida utilizando-se um aparelho Hewlett Packard 5890 Series II equipado com detector de ionização de chama (FID) e uma coluna capilar de sílica fundida J \& W Scientific DB-5 (30 m x 0,25 mm x $0.25 \mu \mathrm{m}$ ); as temperaturas do injetor e detector foram, respectivamente, 220 e $285{ }^{\circ} \mathrm{C}$. Usou-se o hélio como gás de arraste, a uma vazão de $1 \mathrm{~mL} / \mathrm{min}$; o programa de temperatura da coluna foi $40{ }^{\circ} \mathrm{C}(1 \mathrm{~min})$ até $220{ }^{\circ} \mathrm{C}$ a $4{ }^{\circ} \mathrm{C} / \mathrm{min} ; 220$ até $280{ }^{\circ} \mathrm{C}$ a $20^{\circ} \mathrm{C} / \mathrm{min}$. Os espectros de massas foram obtidos com um impacto eletrônico de $70 \mathrm{eV}, 0,84 \mathrm{scan} / \mathrm{sec}$ de m/z 40 a 550 . O hidrogênio foi usado como gás de arraste, com velocidade de fluxo $1,5 \mathrm{~mL} / \mathrm{min}$, modo split (1:10). Uma solução de $1,5 \mu \mathrm{L}$ com $10 \mathrm{mg}$ de óleo em acetato de etila foi injetada. Os índices de retenção foram obtidos pela co-injeção do óleo com uma mistura de hidrocarbonetos lineares $\mathrm{C}_{11}-\mathrm{C}_{24}$ e calculados de acordo com a equação de van den Dool e $\mathrm{Kratz}^{23}$. Os compostos foram identificados com base na comparação dos índices de retenção calculados com os disponíveis na literatura ${ }^{24}$, seguida pela comparação do fragmentograma de padrões das massas relatados na literatura ${ }^{24}$, bem como pela comparação direta das sugestões das massas disponíveis na biblioteca do computador (Wiley, com 250.000 compostos), contemplando apenas as similaridades entre os fragmentogramas.

\section{Criação do ácaro}

O ácaro rajado T. urticae utilizado para os bioensaios foi obtido da criação mantida em plantas de feijão-de-porco (Canavalia ensiformes
L.) do Laboratório de Acarologia Agrícola, do Depto. de Agronomia da UFRPE, a $25 \pm 1^{\circ} \mathrm{C}$, U.R. $65 \pm 3 \%$ e fotofase de $12 \mathrm{~h}$.

\section{Teste de fumigação dos óleos essenciais}

A metodologia para avaliar a ação fumigante dos óleos essenciais sobre os ácaros foi adaptada de Aslan et al. ${ }^{25}$. Recipientes de vidro com capacidade de 2,5 L foram usados como câmaras de fumigação. Três discos de folha de feijão-de-porco $(2,5 \mathrm{~cm})$ foram colocados sobre discos de papel de filtro saturados com água, dentro de placas de Petri de vidro $(9 \mathrm{~cm})$. Em cada disco de folha foram colocadas 10 fêmeas adultas de ácaro-rajado. Cada placa de Petri, contendo no total 30 ácaros, foi colocada no interior de um recipiente de vidro fechado. Os óleos essenciais foram aplicados, com auxílio de pipeta automática, em tiras de papéis de filtro $(5 \mathrm{x}$ $2 \mathrm{~cm}$ ) presas à superfície inferior da tampa dos recipientes. As doses aplicadas foram de 5, 10, 15, 20 e $25 \mu \mathrm{L}$ de cada óleo essencial, o que corresponde a uma concentração de 2, 4, 6, 8 e $10 \mu \mathrm{L} / \mathrm{L}$ de ar, respectivamente. Nada foi aplicado na testemunha. O período de exposição aos óleos foi de 24, 48 e 72 h. Para cada dose e tempo de exposição, 3 repetições foram feitas, sendo cada repetição um recipiente de vidro contendo uma placa de Petri com 30 ácaros.

As avaliações foram feitas ao final do período de exposição. Consideraram-se mortos os ácaros incapazes de caminhar uma distância superior ao comprimento de seu corpo após um leve toque com pincel de cerdas finas. A fecundidade foi avaliada pela contagem do número de ovos. O delineamento deste experimento foi inteiramente casualisado e os dados obtidos foram submetidos à análise de variância com médias comparadas pelo teste de Tukey $(P=0,05)$, calculado pelo Software Sanest 3.0. O método probitos de análise foi usado para obtenção dos valores da concentração letal média $\left(\mathrm{CL}_{50}\right)$ e dos respectivos intervalos de confiança com $95 \%$ de probabilidade ${ }^{26}$.

\section{RESULTADOS E DISCUSSÃO}

Os óleos essenciais obtidos a partir de frutos e folhas de $X$. sericea por meio de hidrodestilação apresentaram uma coloração amarelada e rendimento de 0,22 e $0,43 \%$, respectivamente, tendo sido identificados 24 compostos (Tabela 1).

Todos os componentes voláteis identificados foram sesquiterpenos e monoterpenos, com predominância desse último no óleo dos frutos $(81,71 \%)$ e sesquiterpenos nas folhas $(94,94 \%)$. Os compostos majoritários encontrados no óleo de frutos foram $\beta$-pineno $(45,59 \%)$ e $\alpha$-pineno $(17,18 \%)$. O óleo de folhas foi majoritariamente constituído por cubenol $(57,43 \%)$, seguido por $\alpha$-epimuurolol $(26,09 \%)$.

Os resultados obtidos para o óleo essencial de $X$. sericea, que ocorre em Pernambuco, foram provenientes de uma simples amostra de um único sítio de coleta, sem levar em consideração as possíveis interações intraespecíficas. Entretanto, o óleo de folhas e frutos analisado neste trabalho apresentou um perfil químico diferente, quando comparado com aqueles relatados na literatura ${ }^{16-19}$. Essa diferença pode ser explicada pela distribuição geográfica das populações estudadas, que crescem sob influência de distintas condições de solo e clima, como também pelo horário de coleta do material a ser extraído. Por essa razão, os resultados obtidos na análise química do óleo essencial desta planta sugerem que a mesma seja um novo quimiotipo que ocorre na região Nordeste, especificamente na floresta de restinga no litoral sul de Pernambuco.

Os valores de $\mathrm{CL}_{50}$ calculados para os óleos essenciais do fruto e folha de $X$. sericea são mostrados na Tabela 2, com seus respectivos intervalos de confiança. A toxicidade do óleo da folha ao ácaro- 
Tabela 1. Percentual dos componentes voláteis de folhas e frutos de Xylopia sericea

\begin{tabular}{lccc}
\hline Composto & I.R. $^{\mathrm{a}}$ & \multicolumn{2}{c}{$(\%)$} \\
& & folhas & frutos \\
\hline$\alpha$-Pineno & 941 & 0,41 & 17,18 \\
Canfeno & 956 & 0,25 & - \\
$\beta$-Pineno & 985 & - & 45,59 \\
Mirceno & 997 & - & 9,13 \\
$o$-Cimeno & 1028 & - & 1,25 \\
$p$-Cimeno & 1031 & 0,19 & - \\
Silvestreno & 1035 & - & 2,63 \\
trans-Pinocarveol & 1143 & - & 2,26 \\
$\alpha$-Terpineol & 1190 & - & 1,05 \\
Mirtenal & 1192 & - & 1,22 \\
Mirtenol & 1196 & - & 1,40 \\
Acetato de isomentila & 1310 & 0,26 & - \\
$\alpha$-Cubebeno & 1356 & - & 1,26 \\
$\alpha$-Copaeno & 1378 & - & 7,47 \\
$\alpha$-Cedreno & 1413 & 2,00 & - \\
$\beta$-Gurjuneno & 1432 & - & 1,25 \\
$\gamma$-Elemeno & 1434 & 3,26 & - \\
Seicheleno & 1455 & 1,36 & - \\
Germacreno-D & 1485 & 1,12 & - \\
$\delta$-Cadineno & 1527 & 1,01 & - \\
$\beta$-Oplopenona & 1608 & 2,67 & - \\
$\beta$-Acorenol & 1637 & - & 2,20 \\
$\alpha$-Epi-Muurolol & 1641 & 26.09 & 2,90 \\
Cubenol & 1647 & 57,43 & - \\
Total & & 96,05 & 96,79 \\
\hline
\end{tabular}

ándice de retenção calculado pela co-injeção de uma série homóloga de n-alcanos usando uma coluna capilar apolar DB-5, 30 m.

rajado foi maior e proporcional ao tempo de exposição quando comparado com o do fruto. O óleo das folhas foi 4; 3,5 e 5 vezes mais tóxico que o óleo dos frutos no tempo de exposição de 24,48 e $72 \mathrm{~h}$, respectivamente (Tabela 2). Por outro lado, os valores da $\mathrm{CL}_{50}$ obtidos para o óleo das folhas e frutos nos diferentes períodos de tempo não diferem entre si, em nível de 5\% de probabilidade.

Tabela 2. Valores de $\left(\mathrm{CL}_{50}\right)$ da ação fumigante do óleo essencial de folhas e frutos de $X$. sericea sobre a mortalidade de T. urticae em três períodos de tempo

\begin{tabular}{|c|c|c|}
\hline \multicolumn{3}{|c|}{$\mathbf{D L}_{50}(\mu \mathbf{L} / \mathbf{L}$ de ar) (Intervalo de confiança de $95 \%)$} \\
\hline $24 \mathrm{~h}$ & $48 \mathrm{~h}$ & $72 \mathrm{~h}$ \\
\hline 6,51 & 6,40 & 4,08 \\
\hline$(4,45-10,11)$ & $(5,83-7,01)$ & $(1,50-6,09)$ \\
\hline \multicolumn{3}{|c|}{ Frutos } \\
\hline 27,16 & 22,84 & 20,60 \\
\hline$(22,98-32,98)$ & $(19,63-26,99)$ & $(17,67-24,39)$ \\
\hline
\end{tabular}

Os óleos testados, também interferiram na fecundidade de $T$. urticae, cujos resultados são mostrados na Tabela 3. As menores quantidades médias de ovos colocados pelos ácaros-fêmeas foram observadas quando submetidos aos vapores dos óleos das folhas (12,3 ovos) e frutos (21,0 ovos) no período de $24 \mathrm{~h}$.

Os monoterpenos são compostos provenientes do metabolismo secundário vegetal, que podem causar interferência tóxica nas fun-
Tabela 3. Ação fumigante do óleo essencial de folhas e frutos de $X$. sericea sobre a fecundidade (Média $\pm \mathrm{EP}^{1}$ ) de $T$. urticae em cinco concentrações e três períodos de tempo

\begin{tabular}{lccc}
\hline $\begin{array}{l}\text { Concentração } \\
(\mu \mathrm{L} / \mathrm{L} \text { de ar) }\end{array}$ & \multicolumn{3}{c}{ Fecundidade $^{2}$ (ovos/repetição $)$} \\
& $24 \mathrm{~h}$ & $48 \mathrm{~h}$ & $72 \mathrm{~h}$ \\
\hline 0 & $217,6 \pm 2,96 \mathrm{aA}$ & $277,6 \pm 8,88 \mathrm{aB}$ & $313,3 \pm 8,42 \mathrm{aC}$ \\
2 & $192,6 \pm 6,17 \mathrm{bB}$ & $121,0 \pm 2,0 \mathrm{bC}$ & $301,6 \pm 8,84 \mathrm{aA}$ \\
4 & $118,0 \pm 4,16 \mathrm{cB}$ & $114,3 \pm 1,66 \mathrm{bcB}$ & $220,0 \pm 1,52 \mathrm{bA}$ \\
6 & $67,6 \pm 5,24 \mathrm{dC}$ & $100,6 \pm 1,76 \mathrm{cdB}$ & $203,3 \pm 3,38 \mathrm{bcA}$ \\
8 & $35,3 \pm 2,40 \mathrm{eC}$ & $87,3 \pm 1,45 \mathrm{deB}$ & $179,6 \pm 1,45 \mathrm{cA}$ \\
10 & $21,0 \pm 1,73 \mathrm{eC}$ & $70,0 \pm 3,46 \mathrm{eB}$ & $125,0 \pm 5,57 \mathrm{dA}$ \\
\hline & & Folhas & \\
& $24 \mathrm{~h}$ & $48 \mathrm{~h}$ & $72 \mathrm{~h}$ \\
\hline 0 & $302,0 \pm 9,65 \mathrm{aA}$ & $332,3 \pm 9,35 \mathrm{aB}$ & $324,6 \pm 27,86 \mathrm{aB}$ \\
2 & $19,6 \pm 0,87 \mathrm{bC}$ & $288,6 \pm 11,98 \mathrm{aA}$ & $239,0 \pm 6,24 \mathrm{bB}$ \\
4 & $14,0 \pm 1,0 \mathrm{bB}$ & $171,0 \pm 11,54 \mathrm{bA}$ & $180,6 \pm 3,38 \mathrm{bcA}$ \\
6 & $11,0 \pm 0,57 \mathrm{bB}$ & $181,0 \pm 11,60 \mathrm{bA}$ & $181,3 \pm 7,69 \mathrm{bcA}$ \\
8 & $13,6 \pm 1,20 \mathrm{bC}$ & $173,0 \pm 2,30 \mathrm{bB}$ & $195,6 \pm 4,48 \mathrm{bcA}$ \\
10 & $12,3 \pm 1,20 \mathrm{bB}$ & $177,3 \pm 2,72 \mathrm{bA}$ & $157,6 \pm 12,15 \mathrm{cA}$ \\
\hline
\end{tabular}

${ }^{1} \mathrm{EP}=$ Erro padrão; ${ }^{2}$ médias seguidas da mesma letra maiúscula na mesma linha e minúscula na mesma coluna, para cada óleo, não diferem entre si pelo teste de Tukey $(P=0,05)$.

ções bioquímicas e fisiológicas em herbívoros ${ }^{27}$. A ação de monoterpenos sobre artrópodes tem sido previamente relatada, sugerindo vários modos de ação, como por ex., inibidor da acetilcolestinerase e do citocromo P450 monoxigenase dependente ou atuando no sistema nervoso octopaminérgico do artrópode ${ }^{28}$.

A análise dos constituintes do óleo essencial de folhas e frutos de $X$. aromatica revelou a presença de componentes reconhecidamente inseticidas, como $\alpha$-pineno, $\alpha$-terpineno e limoneno ${ }^{20}$. Em outra investigação, foi atribuída à mistura dos monoterpenos $\alpha$ - e $\beta$-pineno como sendo os componentes ativos do líquido exsudado de uma espécie do gênero Stylosanthes contra as larvas do carrapato-do-boi Boophilus microplus (Canestrini) ${ }^{29}$. Apesar dos monoterpenos $\alpha$ - e $\beta$ pineno juntos somarem mais de $62 \%$ do óleo dos frutos de $X$. sericea, foi o óleo das folhas que se revelou mais ativo contra T. urticae, com valor da $\mathrm{CL}_{50}$ de $4,08 \mu \mathrm{L} / \mathrm{L}$ de ar em $72 \mathrm{~h}$, cujos componentes principais identificados foram os sesquiterpenos oxigenados cubenol $(57,43 \%)$ e $\alpha$-epi-muurolol $(26,09 \%)$. Esse resultado sugere que ácaros e insetos pragas respondem de forma diferente quando submetidos aos componentes químicos de um óleo essencial, ou seja, enquanto que o óleo do fruto constituído com mais de $62 \%$ de $\alpha$ - e $\beta$-pineno revelou menor toxicidade ao ácaro-rajado, com valor da $\mathrm{LC}_{50}$ de 20,60 $\mu \mathrm{L} / \mathrm{L}$ de ar em $72 \mathrm{~h}$, o óleo de Thymus vulgaris constituído majoritariamente por timol $(48,2 \%), \alpha$-pineno $(8,4 \%)$ e $\beta$-pineno $(4,3 \%)$ apresentou forte ação fumigante contra a mosca do cogumelo solani (Lycoriella mali) e, com base nos valores da $\mathrm{CL}_{50}$, os monoterpenos $\alpha$-pineno $(9,85 \mu \mathrm{g} / \mathrm{mL})$ e $\beta$-pineno $(11,85 \mu \mathrm{g} / \mathrm{mL})$ foram os responsáveis pela toxicidade ${ }^{30}$. Por outro lado, não se deve excluir o possível efeito sinérgico de outros constituintes presentes no óleo do fruto, que mesmo em pequenas quantidades podem ter mascarado a reconhecida atividade acaricida de ambos os monoterpenos bicíclicos descritos anteriormente por Sutherst et al..$^{29}$ e Choi et al..$^{30}$.

\section{AGRADECIMENTOS}

À CAPES pela concessão de bolsa de mestrado para W. J. T. Pontes, e ao CNPq, pela bolsa de Iniciação Científica e suporte financeiro. 


\section{REFERÊNCIAS}

1. Viegas Júnior, C.; Quim. Nova 2003, 26, 390.

2. Hernández, C. R.; Plantas contra Plagas - potencial práctico de ajo, anona, nim, chile y tabaco, RAPAM: Estado de México, 2001.

3. Moreira, I. C.; Lago, J. H. G.; Roque, N. F.; Biochem. Syst. Ecol. 2005, 33 948.

4. Nishiyama, Y.; Moriyasu, M.; Ichimaru, M.; Iwasa, K.; Kato, A.; Mathenge, S. G.; Mutiso, P. B. C.; Juma, F. D.; Phytochemistry 2004, 65, 939.

5. De-Andrade, N. C.; Barbosa-Filho, J. M.; Da-Silva, M. S.; Da-Cunha, E. V. L.; Maia, J. G. S.; Biochem. Syst. Ecol. 2004, 32, 1055.

6. Stashenko, E. E.; Jaramillo, B. E.; Martinéz, J. R.; J. Chromatogr., A 2004, 1025, 105.

7. Tatsadjieu, L. N.; Essia-Ngang, J. J.; Ngassoum, M. B.; Etoa, F. X.; Fitoterapia 2003, 74, 469.

8. Konning, G. H.; Agyane, C.; Ennison, B.; Fitoterapia 2004, 75, 65.

9. Asekun, O. T.; Kunle, O.; J. Essent. Oil-Bearing Plant. 2004, 7, 186.

10. Alexander, I. C.; Pascoe, O. K.; Marchand, P.; Williams, L. A. D.; Phytochemistry 1991, 30, 1801.

11. Lajide, L.; Escoubas, P.; Mizutani, J.; Phytochemistry 1995, 40, 1105.

12. Okonkwo, E. U.; Okoye, W. I. I.; J. Pest. Manag. 1996, 42, 143.

13. Ngamo, L. S. T.; Ngassoum, M-B.; Jirovetz, L.; Ousman, A.; Nukenine, E. C.; Mukala, O. E.; Mededelingen - Faculteit Landbouwkundige en Toegepaste Biologische Wetenschappen, 2001, 66, 473.

14. Corrêa, M. P.; Dicionário das Plantas Úteis do Brasil e das Exóticas Cultivadas, Imprensa Nacional: Rio de Janeiro, 1987, vol. 5.
15. Takahashi, J. A.; Henrieta, S. V.; Boaventura, M. A. D.; Quim. Nova 2001, 24, 616.

16. Craveiro, A. A.; Alencar, J. W.; Nosttrowsy, O.; J. Nat. Prod. 1986, 49, 1146.

17. Lemos, T. L. G.; Monte, F. J. Q.; Matos, F. J. A.; Alencar, J. W.; Craveiro, A. A.; Barbosa, R. C. S. B.; Lima, E. O.; Fitoterapia 1992, 63, 266.

18. Camara, C. A. G.; Alencar, J. W.; Silveira, E. R.; J. Essent. Oil Res. 1996, 8, 75.

19. Fournier, G.; Hadjiakhoondi, A.; Leboeuf, M.; Cave, A.; Charles, B.; Fourniat, J.; Phytotherapy Res. 1994, 8, 166

20. Potenza, M. R.; Takematsu, A. P.; Sivieri, A. P.; Sato, M. E.; Passerotti, C. M.; Arq. Inst. Biol. 1999a, 66, 31

21. Potenza, M. R.; Takematsu, A. P.; Benedicto, L. H.; Arq. Inst. Biol. 1999b, 66,91

22. Robinson-Vargas, M.; Chapman, B.; Pearman, D. R.; Agric. Tec. 2001, 61, 3.

23. van den Dool, H.; Kratz, P. H.; J. Chromatogr., A 1963, 11, 463.

24. Adams, R. P.; Identification of Essential oil Components by Gas Chromatography/Mass Spectroscopy, Allured Publ. Corp.: Carol Stream, 1995.

25. Aslan, I.; Özbek, H.; Calmasur, Ö.; Sahin, F.; Ind. Crop Prod. 2004, 19, 167.

26. Finney, D. J.; Probit Analysis, A statistical Treatment of the Sigmoid Response Curve, University Press: Cambridge, 1974.

27. Dunkel, F. V.; Sears, L. J.; J. Stor. Prod. Res. 1998, 34, 307.

28. De-Oliveira, A. C.; Ribeiro-Pinto, L. F.; Paumgartten, J. R.; Toxicol. Lett. 1997, 92, 39; Enam, E. E.; Annual Meeting of the Entomological Society of America, San Diego, USA, 2001; Ryan, M. F.; Byrne, O.; J. Chem. Ecol. 1988, 14, 1965.

29. Sutherst, R. W.; Jones, R. J.; Schnitzerling, H. J.; Nature 1982, 295, 320.

30. Choi, W. S.; Park, B. S.; Lee, Y. H.; Jang, D. Y.; Yoon, H. Y.; Lee, S. E.; Crop Prot. 2006, 25, 398 